\title{
4 Editorial Remarks
}

The following texts were transcribed from the Ernst Papanek Papers at the New York Public Library, Box 8 (Writings by Ernst Papanek on refugee children), but are not presented as a critical text edition. The single texts are simply reproduced as the original ones, although minor typos and mistakes have been silently corrected. Additions are clearly marked with brackets [], while omissions have been marked with [. . . ]. If the text as such needed further information or contextualization, explanatory footnotes were added. Page numbers are presented according to the original texts in parenthesis as well. 